\title{
EL POSICIONAMIENTO EN REDES SOCIALES DE LAS INSTITUCIONES PÚBLICAS DEL ECUADOR.
}

\section{THE POSITIONING IN SOCIAL NETWORKS OF THE PUBLIC INSTITUTIONS OF ECUADOR.}

Víctor Eduardo Romero Cueva, Econ. Economista con mención en Gestión Empresarial (Ecuador). Estudiante de la Maestría en Economía con mención en Desarrollo Económico y Políticas Públicas de la Universidad Tecnológica ECOTEC (Ecuador). Docente del Curso de Nivelación de Carrera en la Universidad Técnica de Machala, Ecuador. viromero@mgs.ecotec.edu.ec

Jorge Luis Benites Suquitana, Ing. Ingeniero de Sistemas (Ecuador). Analista de Gestión, Mejoramiento, Evaluación Académica y Titulación en la Universidad Técnica de Machala, Ecuador. jbenites@utmachala.edu.ec

\section{Alfonso Iván Fuentes Díaz, Econ.}

Economista con Gestión Empresarial (Ecuador). Estudiante de la Maestría en Economía con mención en Desarrollo Económico y Políticas Públicas de la Universidad Tecnológica ECOTEC (Ecuador). Administrador de Locales Comerciales, Ecuador. alofuentes@mgs.ecotec.edu.ec

Rafael Armando Largo Valdiviezo, Ing. Ingeniero en Administración de Empresas con énfasis en Marketing (Ecuador). Estudiante de la Maestría en Economía con mención en Desarrollo Económico y Políticas Públicas de la Universidad Tecnológica ECOTEC (Ecuador). Analista de Gestión Tributaria en Servicio de Rentas Internas (SRI), Ecuador.

\section{ARTÍCULO DE REFLEXIÓN}

largo@mgs.ecotec.edu.ec

Recibido: 4 de septiembre de 2018.

Aceptado: 30 de noviembre de 2018. 


\section{RESUMEN}

La información actualmente se ha convertido en un activo muy importante para las empresas tanto públicas como privadas y las redes sociales son de vital importancia. Por esta razón, única condición necesaria y suficiente para que una empresa pública tenga presencia online es que divulgue información pertinente a la ciudadanía. En tal sentido es posible que el contenido publicado no alcance la audiencia objetiva, aunque la comunicación hoy día es más accesible e inmediata, es posible que el contenido publicado no alcance la audiencia objetivo. El presente estudio analiza las estrategias digitales aplicadas por las instituciones públicas del Ecuador y cómo estas afectan su posicionamiento en redes sociales. Para ello, se utilizó el método de observación y el software online Social Bakers como instrumento de análisis. A su vez, fueron seleccionadas 6 instituciones públicas con presencia online afines al sector productivo y comercial. Adicionalmente, se identificó las plataformas más utilizadas a nivel nacional. En este caso, se analizó el contenido generado y se midió el impacto en la audiencia de Facebook, Twitter y YouTube. Los resultados evidenciaron que la mayoría de las instituciones analizadas no han logrado posicionarse virtualmente por diferentes motivos presentes en la investigación.

Palabras clave: posicionamiento online, redes sociales, instituciones públicas, inbound marketing, contenido.

\section{ABSTRACT}

The information currently has become a very important asset for both public and private companies and social networks are of vital importance. For this reason, the only necessary and sufficient condition for a public company to have an online presence is to disclose pertinent information to the people. In this regard, it is possible that the published content does not reach the objective audience, although, today communication is more accessible and immediately, it is possible that the published content does not reach the target audience. The present study analyzes the digital strategies applied by the public institutions of Ecuador and how these affect their social network positioning. For this, the method of observation and the online software Social Bakers was used as an instrument of analysis. At the same time, 6 public institutions with an online presence related to the productive and commercial sector were selected. Additionally, the most used platforms nationwide were identified. In this case, the content generated was analyzed and the impact on the audience of Facebook, Twitter and YouTube 
was measured. The results showed that most of the analyzed institutions have not been able to position themselves for different reasons present in the research.

Keywords: online positioning, social networks, public institutions, inbound marketing, content

\section{INTRODUCCIÓN}

La historia registra varios descubrimientos e inventos que cambiaron el curso de la humanidad. Las revoluciones industriales aceleraron el proceso de transformación social, económica y tecnológica en el mundo. Gracias a ello, la vida cotidiana se volvió más confortable, puesto que muchas labores que antes tomaban bastante tiempo en efectuar se redujeron notablemente.

La información se ha venido transmitiendo de generación en generación por cada una de las civilizaciones, esto ha sido determinante para facilitar la creación e innovación. De hecho, la llegada del Internet en el siglo pasado, abrió las puertas hacia el espacio virtual y, consecuentemente, la universalización de la información. Independientemente de su clase social, etnia o género, todos pueden acceder a ella. Es decir, no hay barreras, horarios ni distancias.

Internet cambió muchas cuestiones, una de ellas, la forma de hacer negocios. Hoy día, la mayoría de las empresas han incursionado en el mundo digital, y no es para menos dado el enorme número de usuarios conectados; que buscan diariamente contenido sobre diversos productos. De hecho, Google se ha convertido en el gran aliado de millones de consumidores, y también vendedores. Por lo que, si no se halla en los resultados de búsqueda el sitio web de alguna empresa, sería como si no existiera.

Asimismo, las redes sociales se han convertido en el canal de comunicación por excelencia. El contenido que se comparte en las distintas plataformas es parte vital de la publicidad. Cuando se crea contenido, no solo se intenta vender un producto, también representa la identidad corporativa. Para Barrientos (2016)1 "la empresa ha cambiado de manera significativa con la introducción de internet: la que no se adapta, sale del mercado" (p.12).

Actualmente casi todas las empresas tienen un sitio web o página oficial, no importa su tamaño. Desde la más pequeña hasta la más grande. Unas utilizan el internet como recurso para posicionar su marca, otras lo hacen solo como medio de contacto. Las primeras, son 
creadoras de contenido y buscan a través de la difusión, atraer más clientes. Araujo (2016, p. 2) lo explica:

Una de las tendencias que determina el éxito empresarial actualmente, es la adaptación a los cambios de los clientes arraigados a la tecnología. El cliente hoy en día se encuentra muy bien informado, le agrada la comodidad, tiene nuevas rutas de compra, carece de tiempo extra y sobre todo no le agrada ser interrumpido. Y si bien resulta ser para muchos una problemática y dicha adaptación, tomarlo como oportunidad resulta interesante. Ya que, si se logra solicitar el permiso o ser un insumo de valor para el cliente, se puede llegar a entablar una relación que se adapta a los niveles, que van desde; desconocido - amigo a cliente perdurable.

En relación con la generación de contenido, las empresas privadas son muy habituales, puesto que cada actualización es un intento para mejorar la experiencia de usuario, y con ello incrementar las ventas de sus productos. De modo similar, las empresas públicas se comportan en la esfera virtual, puesto que su presencia online en redes sociales genera beneficios en cuanto a cercanía con los ciudadanos, atención a sus problemas y necesidades; así como la divulgación de actividades derivadas de la gestión pública. Dicho sea de paso, permite una constante comunicación y tiene un gran poder de difusión social.

En el caso particular de Ecuador, el Gobierno Nacional, a través del Ministerio de Telecomunicaciones y de la Sociedad de la Información MINTEL, pretende contribuir en la consolidación del desarrollo local y productivo, a través del diálogo constante, con la ayuda de los diferentes sectores del país. En otras palabras, la información proporcionada por las empresas públicas es una condición importante para fortalecer la participación de las diversas entidades públicas, privadas, academia y, sobre todo, de la sociedad civil.

En efecto, uno de los ejes planteados por el gobierno ecuatoriano se refiere a la inclusión digital. Ecuador es un país con 16.74 millones de habitantes, según el portal Hootsuite (2018), hay 13.47 millones de usuarios de internet, equivalente a un $80 \%$. 


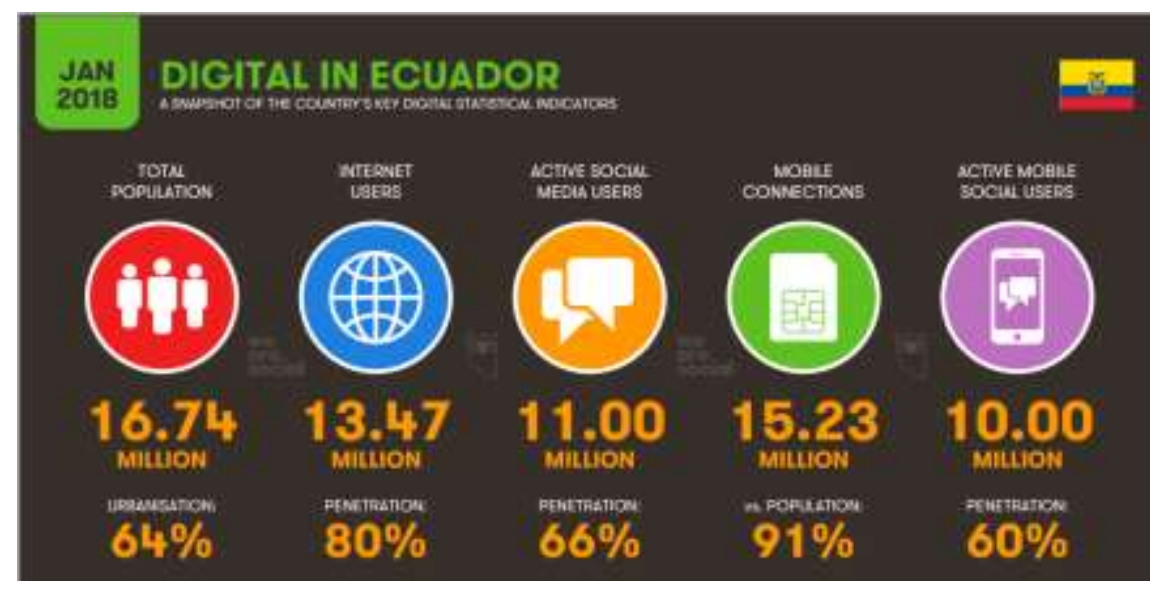

Figura 1: Población de Ecuador.

Fuente: Hootsuite.

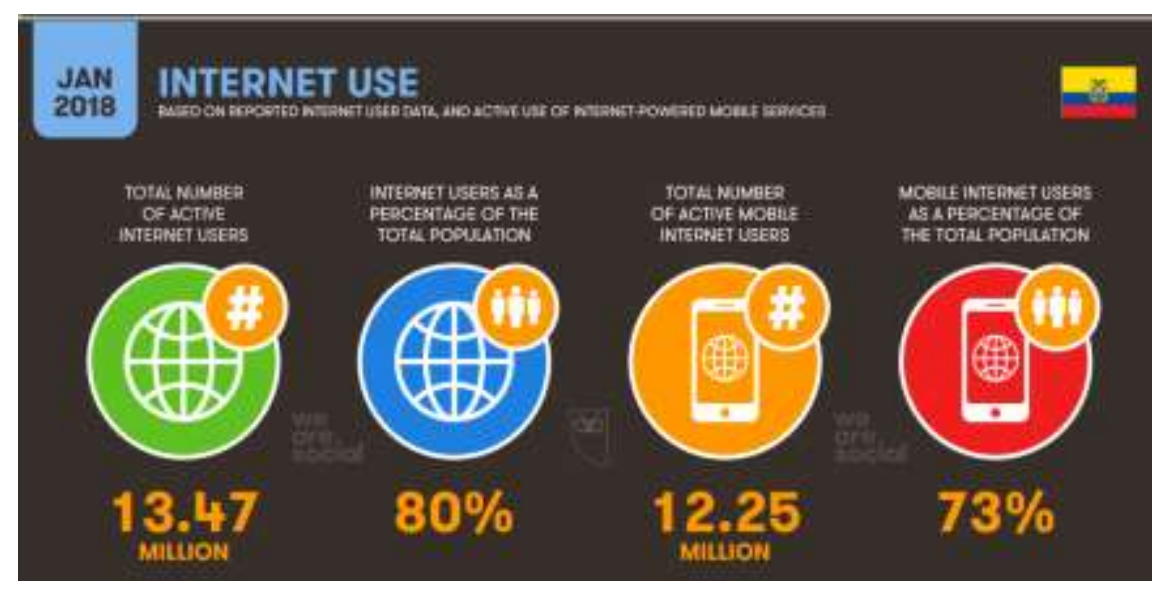

Figura 2: Población de Ecuador.

Fuente: Hootsuite.

También hay 11 millones de usuarios activos en redes sociales, lo que equivale al $66 \%$. Entonces, no se deduce otra cosa, sino que la mayoría de la población tiene acceso a la información digital. 


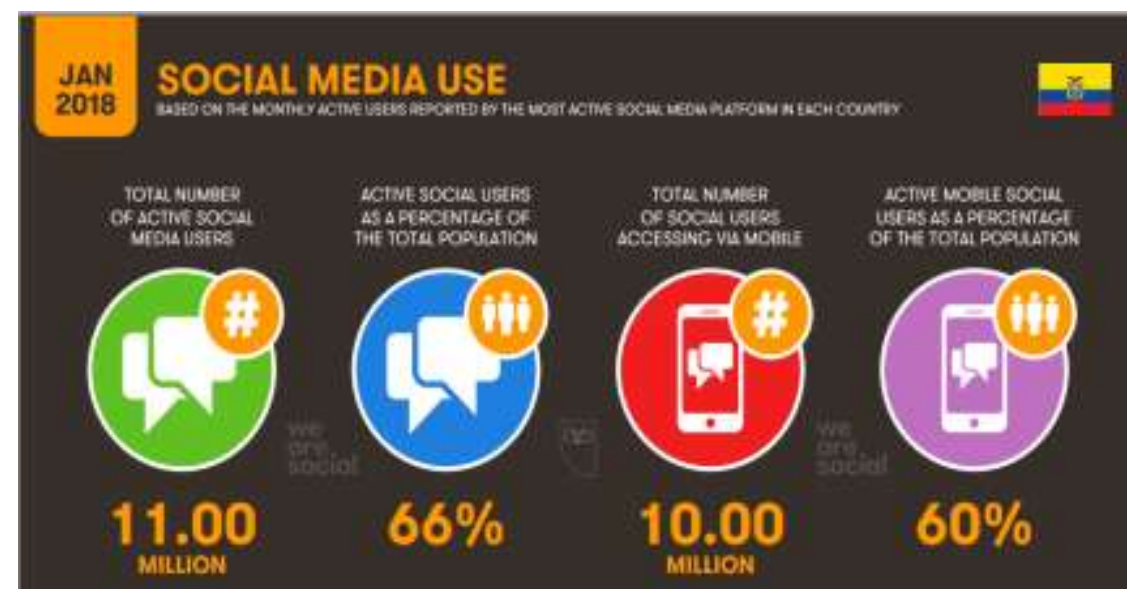

Figura 3: Población de Ecuador.

Fuente: Hootsuite.

Ahora bien, el quid de la cuestión es: ¿qué sitios web están visitando los ecuatorianos? El Ranking Alexa (2018), presenta una lista encabezada por Google Ecuador, en segundo lugar, aparece YouTube, continuando con Google (el buscador clásico), en cuarto lugar, Facebook, entre otros. Como dato adicional, el Instituto Ecuatoriano de Seguridad Social (IESS) ha sido la única entidad pública dentro del top 20. Y no es para menos, dado el enorme potencial de difusión que este portal posee, sobre todo, por las diversas prestaciones sociales que brinda.

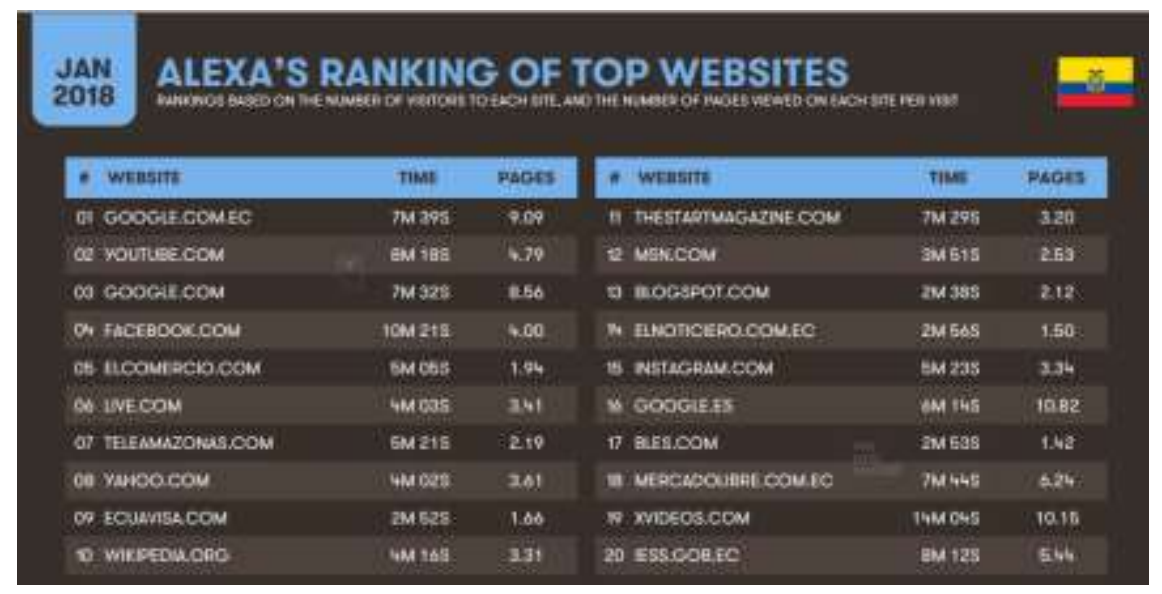

Figura 4: Alexa.

Fuente: Hootsuite. 
De modo similar, referido a los términos más ingresados en Google, quien encabeza la lista es Facebook, mientras que IESS ocupa la casilla número 15. Google Trends (2018).

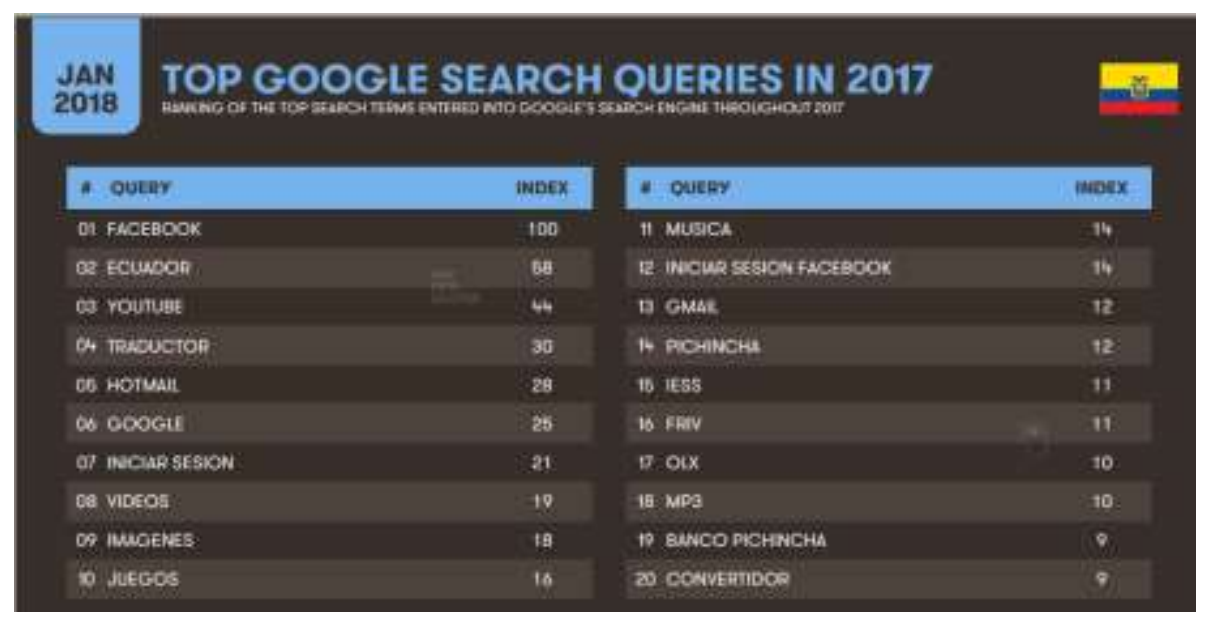

Figura 5: Google Trends

Fuente: Hootsuite.

Por otro lado, hay que destacar que existen ciertas instituciones públicas de Ecuador que presentan un bajo posicionamiento en sus sitios web, especialmente, aquellas que están vinculadas directamente con el escenario productivo y comercial. Si bien cierto, gran parte de los ecuatorianos tienen acceso a internet, muy pocos lo utilizan para visitar sitios de carácter informativo. Resulta irónico el hecho de que en plena era de la información, haya tanta gente desinformada. Difícilmente sea una cuestión de recursos, gracias a la globalización el número de personas conectadas se encuentra en constante crecimiento.

Tal vez, la forma cómo las empresas públicas generan contenido en redes sociales sea un factor explicativo. Hay un indicador que lo confirma, ninguna o pocas reacciones en las publicaciones hechas por determinadas cuentas del Gobierno Central. Por si fuera poco, los problemas derivados de la gestión en páginas institucionales están impactando fuertemente en la comunicación. Cabe destacar que cada institución posee programas dirigidos a sectores específicos de la economía, los cuales son diseñados con el fin de generar beneficios a los involucrados.

Pero, ¿qué sucede cuando la población no se entera de la existencia de estos programas? Ciertamente, si una publicación de carácter informativo no tiene el alcance deseado, los resultados saltan a la vista: no se cumplen los objetivos institucionales, esto es, la sociedad 
civil no se empodera de los recursos, lo cual desencadena en un escaso desarrollo económico.

Anteriormente se evidenció el potencial que tiene Facebook como medio de comunicación, sin embargo, hay otras plataformas como Twitter o Instagram que bien podrían ser utilizadas para facilitar la experiencia de usuario en Ecuador.

Últimamente, la aplicación del Inbound Marketing ha sido pieza clave en el posicionamiento web de las empresas privadas, dado que facilita el análisis de datos y la toma de decisiones. Así pues, dicha aplicación se presenta como una oportunidad para las instituciones públicas del Ecuador y su vinculación con la comunidad.

\section{Formulación del Problema:}

¿Cuáles son los factores más influyentes para posicionar las instituciones públicas del Ecuador en redes sociales?

\section{Objetivo general:}

Identificar los factores más influyentes de posicionamiento en redes sociales de las instituciones públicas del Ecuador para mejorar la divulgación de la información.

\section{Objetivos específicos:}

- Medir el posicionamiento en redes sociales de las instituciones públicas del Ecuador

- Identificar oportunidades que mejoren la experiencia de usuario en las redes sociales utilizadas por las instituciones públicas del Ecuador

- Aplicar estrategias que aumenten la visibilidad y posicionamiento en redes sociales de las instituciones públicas del Ecuador

\section{REVISIÓN TEÓRICA}

\subsection{Redes Sociales en Instituciones Públicas.}

Realizar una definición de las redes sociales en las administraciones públicas no es sencillo. La nueva manera de actuar se destaca en la transparencia, la participación, la rendición de cuentas, etc., como nuevos principios de funcionamiento. Así lo señalan diferentes autores de referencia en la materia (Bonson, Criado y Rojas Martín, Mergel, \& Bretschneider, 2017). Básicamente, las entidades públicas utilizan las redes sociales para divulgar información, cuya audiencia principal es la sociedad civil. 
Cuadro 1. Cambios hacia una Administración Pública 2.0

\begin{tabular}{|c|c|c|}
\hline & $\begin{array}{c}\text { Administración pública } \\
\text { tradicional }\end{array}$ & Administración 2.0 \\
\hline $\begin{array}{l}\text { Objetivo básico } \\
\text { de funciona- } \\
\text { miento }\end{array}$ & $\begin{array}{l}\text { Previsión de servicios en lí- } \\
\text { nea de carácter transaccional }\end{array}$ & $\begin{array}{l}\text { Emergencia de iniciativas } \\
\text { (bottom-up) compartidas con el } \\
\text { ciudadano/empleado público } \\
\text { como eje central }\end{array}$ \\
\hline $\begin{array}{l}\text { Mecanismo de } \\
\text { retroalimenta- } \\
\text { ción }\end{array}$ & $\begin{array}{l}\text { Cuestionarios a grupos de } \\
\text { discusión poco frecuentes y } \\
\text { con pocos clientes/ciudada- } \\
\text { nos de la organización }\end{array}$ & $\begin{array}{l}\text { Comentarios continuos proce- } \\
\text { dentes de los miembros de la } \\
\text { comunidad }\end{array}$ \\
\hline $\begin{array}{l}\text { Interacciones } \\
\text { con el público }\end{array}$ & $\begin{array}{l}\text { Baja interacción; unidireccio- } \\
\text { nales }\end{array}$ & $\begin{array}{l}\text { Alta interacción; bidirecciona- } \\
\text { les }\end{array}$ \\
\hline $\begin{array}{l}\text { Procesos de } \\
\text { adopción de } \\
\text { decisiones }\end{array}$ & Decisiones internas & Gobernanza compartida \\
\hline Público objetivo & $\begin{array}{l}\text { Orientación a audiencias } \\
\text { masivas: el público }\end{array}$ & $\begin{array}{l}\text { Audiencias personalizadas y } \\
\text { bien segmentadas }\end{array}$ \\
\hline Medición & $\begin{array}{l}\text { Puramente cuantitativa, con } \\
\text { un acceso limitado a com- } \\
\text { portamientos e identidades } \\
\text { de los individuos }\end{array}$ & $\begin{array}{l}\text { Datos en tiempo real, masivos, } \\
\text { con un potencial de análisis } \\
\text { cualitativo sobre opiniones y } \\
\text { actitudes, así como de elemen- } \\
\text { tos socio-demográficos }\end{array}$ \\
\hline
\end{tabular}

Fuente: Gil, Criado, \& Téllez (2017)

Las nuevas herramientas sociales admiten participación colaborativa por parte de los ciudadanos dentro de los espacios públicos de las administraciones públicas en internet, asumiendo que las intervenciones externas son importantes para nutrir los perfiles en Facebook, YouTube y otras redes sociales.

Las interacciones con la ciudadanía aportan potencialmente la eficiencia económica, ya no es necesaria la presencia física en los edificios gubernamentales, la prestación del servicio público se puede producir en cualquier momento, de forma inmediata y con una segmentación mucho más precisa de las necesidades atendidas.

\subsection{Posicionamiento en redes sociales.}

Hasta hace poco, el posicionamiento de una empresa se enfocaba principalmente en llegar a los usuarios a través de herramientas tradicionales de mercadotecnia, y con ello, difundir las bondades de determinado producto para incrementar las ventas y mejorar la popularidad frente a la competencia. En la actualidad, a pesar de los cambios constantes, el objetivo se mantiene, pero los medios utilizados tienen un nuevo entorno. $Y$ no solo para las empresas privadas sino también para las públicas. El entorno virtual, específicamente las plataformas online conocidas como redes sociales, han llegado a ser el medio de comunicación por excelencia. El consumidor, hoy llamado audiencia, recepta información divulgada por las 
instituciones públicas, otras veces no. Cuando la información publicada tiene un largo alcance e impresión, la visibilidad aumenta y consecuentemente, el posicionamiento.

\subsection{Inbound Marketing.}

Para Andrade (2016), son todas las estrategias de Marketing en donde "No tienes que pagar". Según explica Internet República en su blog, el inbound marketing se basa en tres pilares fundamentales: SEO, marketing de contenidos y social media marketing. Ya sea en redes sociales como Twitter, en Facebook con la creación de páginas empresariales, YouTube.

El Inbound Marketing, consiste en hacer que sea el usuario quien te encuentre, en lugar de perseguirlo y molestarlo como ocurre en la publicidad habitual.

\section{MATERIALES Y MÉTODOS}

Se basó en el método de observación, el cual estuvo apoyado en la utilización de SocialBakers, una herramienta gratuita para el análisis de plataformas online, principalmente, redes sociales. Así pues, se extrajo información sobre las siguientes métricas: distribución de las interacciones, distribución del tipo de publicación y tipos de publicaciones más interesantes.

Las plataformas analizadas fueron seleccionadas considerando la pertinencia de cada una de ellas dentro del sector productivo y comercial, tal es el caso de:

Instituto de Economía Popular y Solidaria, Instituto de Promoción de Exportaciones e Inversiones del Ecuador Pro Ecuador Ministerio de Agricultura y Ganadería, Ministerio de Industrias y Productividad Mipro, Ministerio de Turismo y Superintendencia de Compañías, Asimismo, las redes sociales objeto de estudio fueron Facebook, Twitter y YouTube.

\section{ANÁLISIS DE LOS RESULTADOS}

\subsection{Análisis de Redes Sociales.}

\section{FACEBOOK}




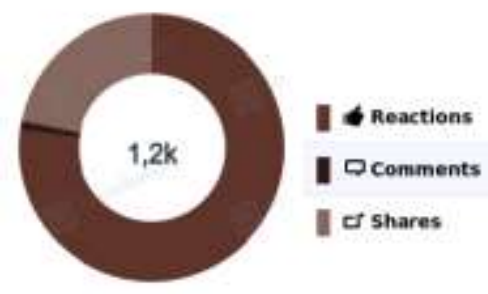

$\begin{array}{lr}\text { cropt } & \text { share } \\ 963 & 77,23 \% \\ 13 & 1,04 \% \\ 271 & 21,73 \%\end{array}$

Distribution of Page Post Types

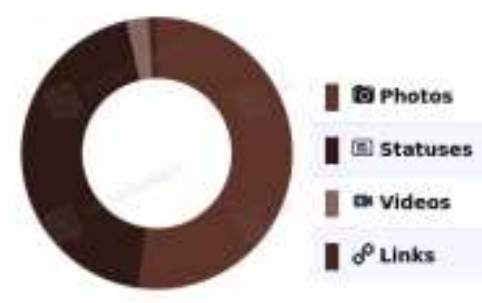

Jul 19, 2018 - Aug 16, 2018

\begin{tabular}{rr|} 
Count & sharm \\
\hline 160 & $52,29 \%$ \\
\hline 135 & $44,12 \%$ \\
\hline 8 & $2,61 \%$ \\
\hline 3 & $0,98 \%$ \\
\hline
\end{tabular}

Jul 19, 2018 - Aug 16, 2018

Most Engaging Post Types

Aug interactiuns per 1000 fans

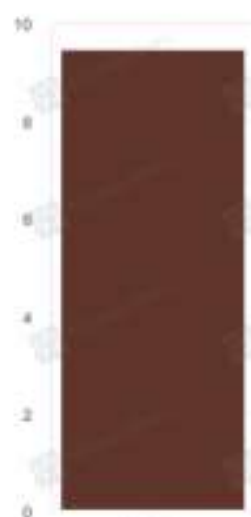

Prote

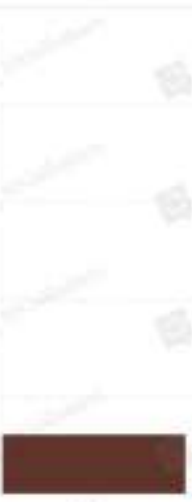

yous

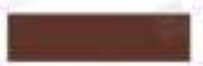

Lan

Figura 6. Facebook/Páginas de Instituciones Públicas del Ecuador.

Fuente: Social Baker \& autores a partir del Instituto de Economía Popular y Solidaria. 
Distribution of Interactions

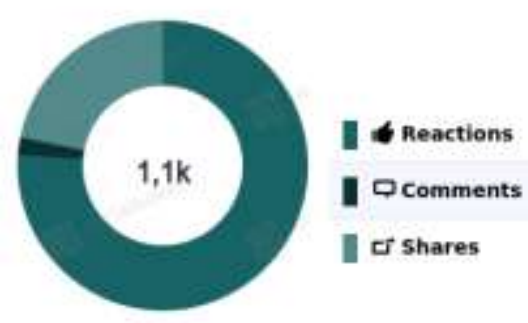

Distribution of Page Post Types

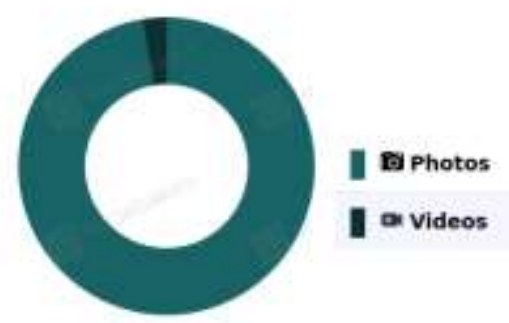

Most Engaging Post Types

Avig interactions per 1000 Fans

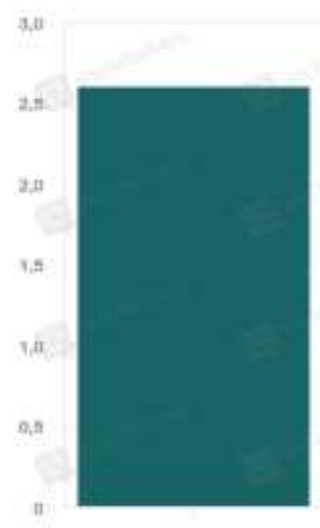

Whoter:

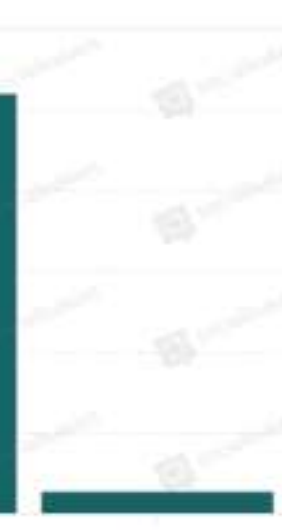

nisos
Jut 19, 2018 - Aug 16, 2018

\begin{tabular}{|c|c|}
\hline Count & Share \\
\hline 820 & $76,21 \%$ \\
\hline 21 & $1.95 \%$ \\
\hline 235 & $21.84 \%$ \\
\hline
\end{tabular}

Jul 19, 2018 - Aug 16, 2018

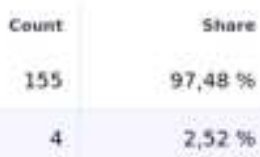

ful 19, 2018 - Aug 16, 2018

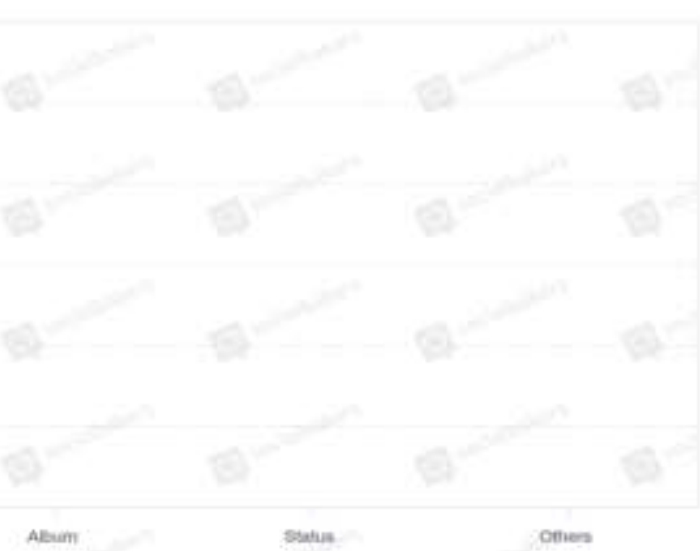

Figura 7. Facebook/Páginas de Instituciones Públicas del Ecuador

Fuente: Social Baker \& autores a partir del Instituto de Promoción de Exportaciones e Inversiones ProEcuador. 

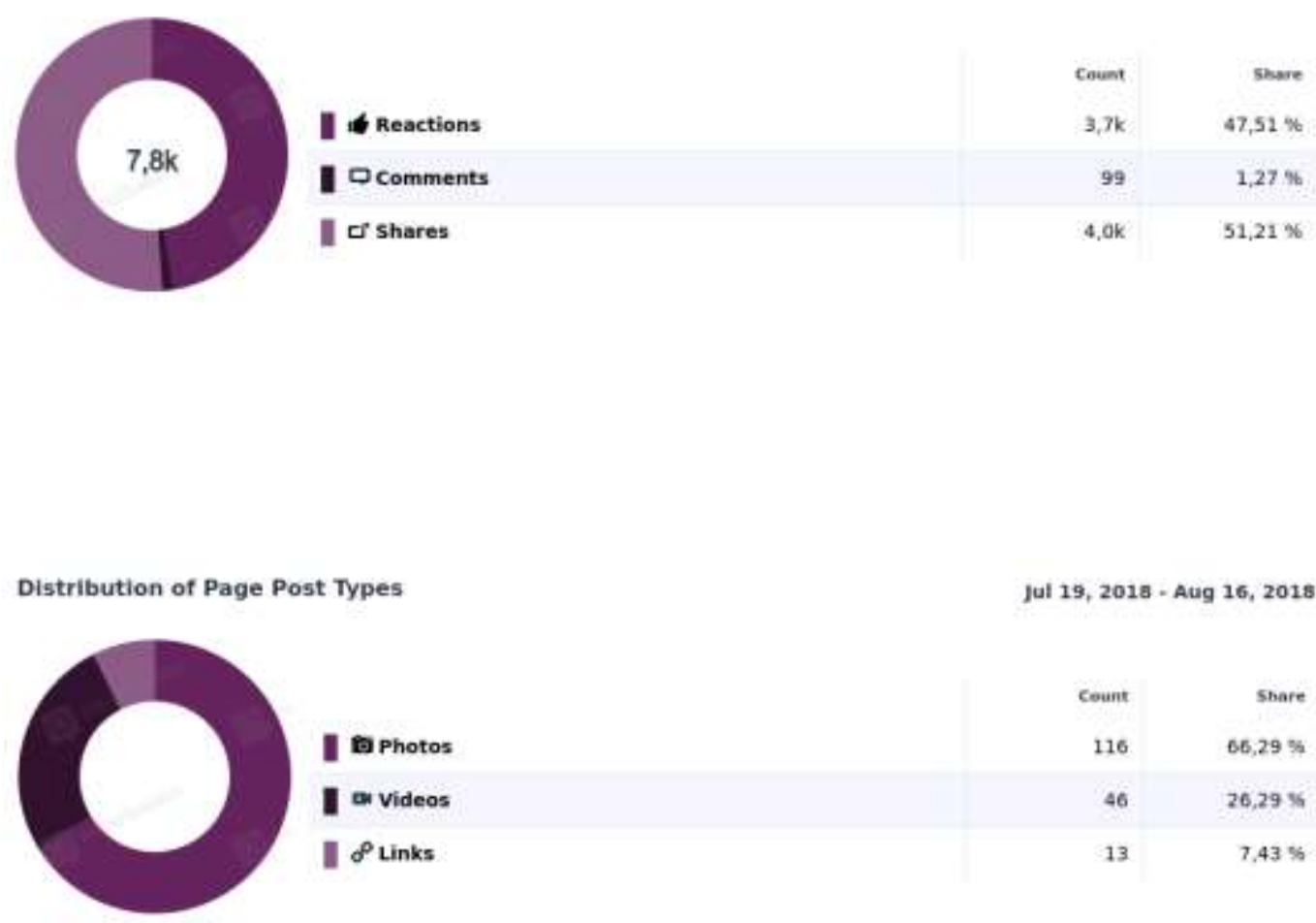

Most Engaging Post Types

Avg Interactions per 1000 Fans

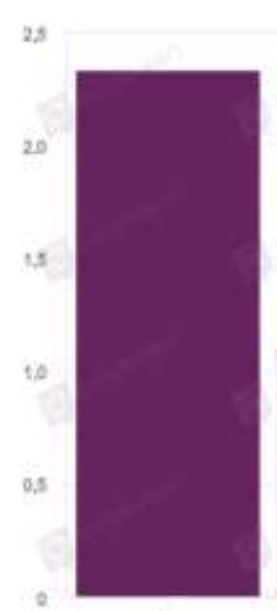

Lis

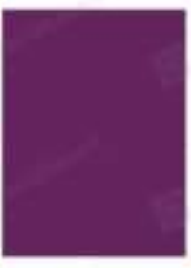

Prote

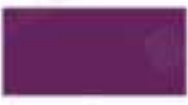

vaseo
Assum ติatus
Chen

Figura 8. Facebook/Páginas de Instituciones Públicas del Ecuador.

Fuente: Social Baker \& autores a partir del Ministerio de Agricultura y Ganadería. 


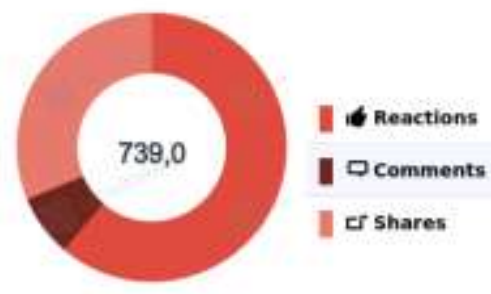

$\begin{array}{rr}\text { Count } & \text { smere } \\ 453 & 61,30 \% \\ 55 & 7.44 \% \\ 231 & 31,26 \%\end{array}$

Distribution of Page Post Types

Jut 19, 2018 - Aug 16, 2018
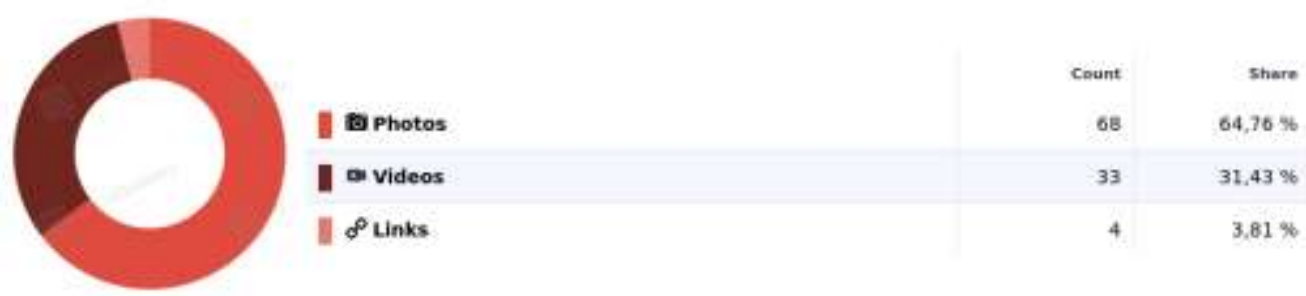

Most Engaging Post Types

Jul 19, 2018-Aug 16, 2018

Aug interactions per 1000 mans

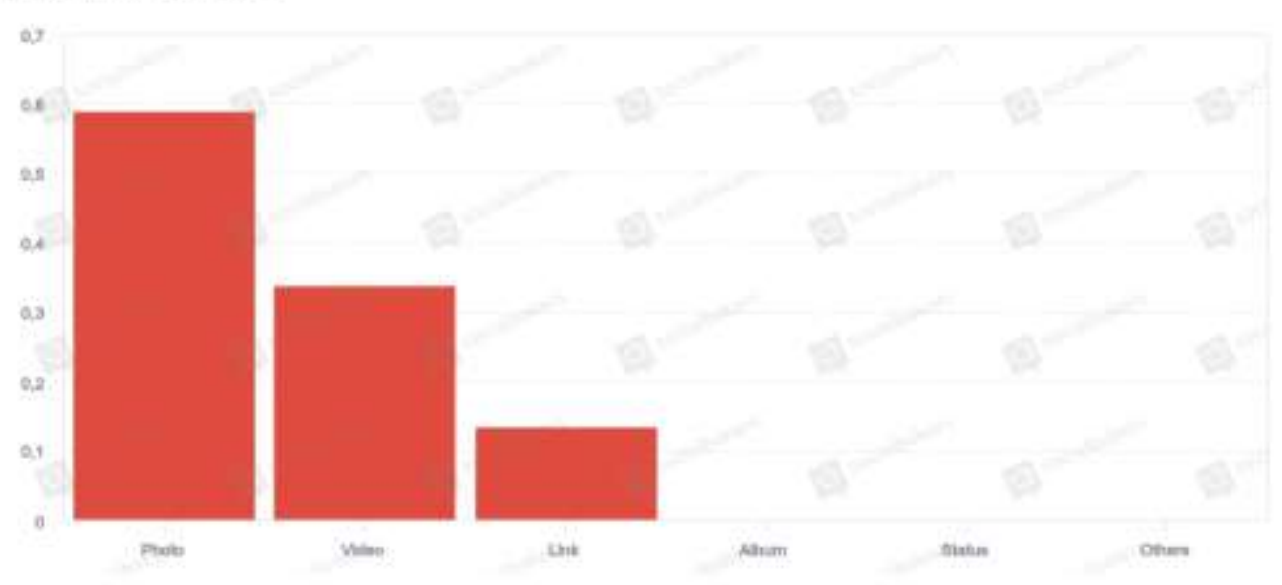

Figura 8. Facebook/Páginas de Instituciones Públicas del Ecuador.

Fuente: Social Baker \& autores a partir del Ministerio de Industrias. 
Distribution of Interactions

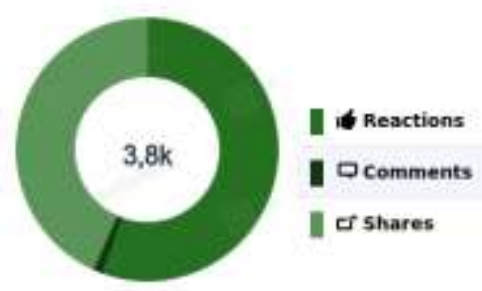

Distribution of Page Post Types

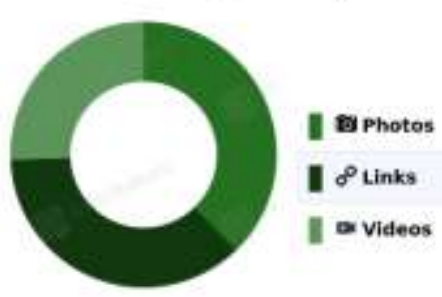

Jul 19, 2018 - Aug 16, 2018

$\begin{array}{rr}\text { Coumt } & \text { Share } \\ 2,1 \mathrm{k} & 55,61 \% \\ 45 & 1,17 \% \\ 1,7 \mathrm{k} & 43,21 \%\end{array}$

Jut 19, 2018 - Aug 16, 2018

$\begin{array}{rr}\text { Count } & \text { share } \\ 31 & 37,80 \% \\ 30 & 36,59 \% \\ 21 & 25.61 \%\end{array}$

ful 19, 2015 - Aug 16, 2018

Most Engaging Post Types

Avg interactions por 1000 fans

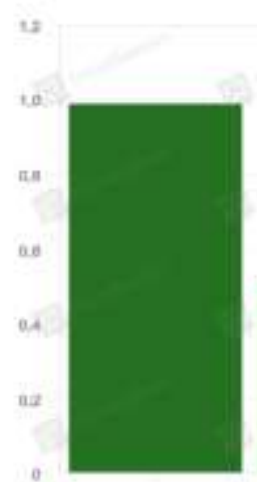

vinu

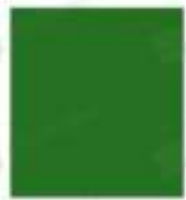

un

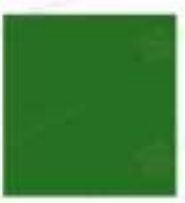

Ptwis

Figura 9. Facebook/Páginas de Instituciones Públicas del Ecuador

Fuente: Social Baker \& autores a partir del Ministerio de Turismo. 

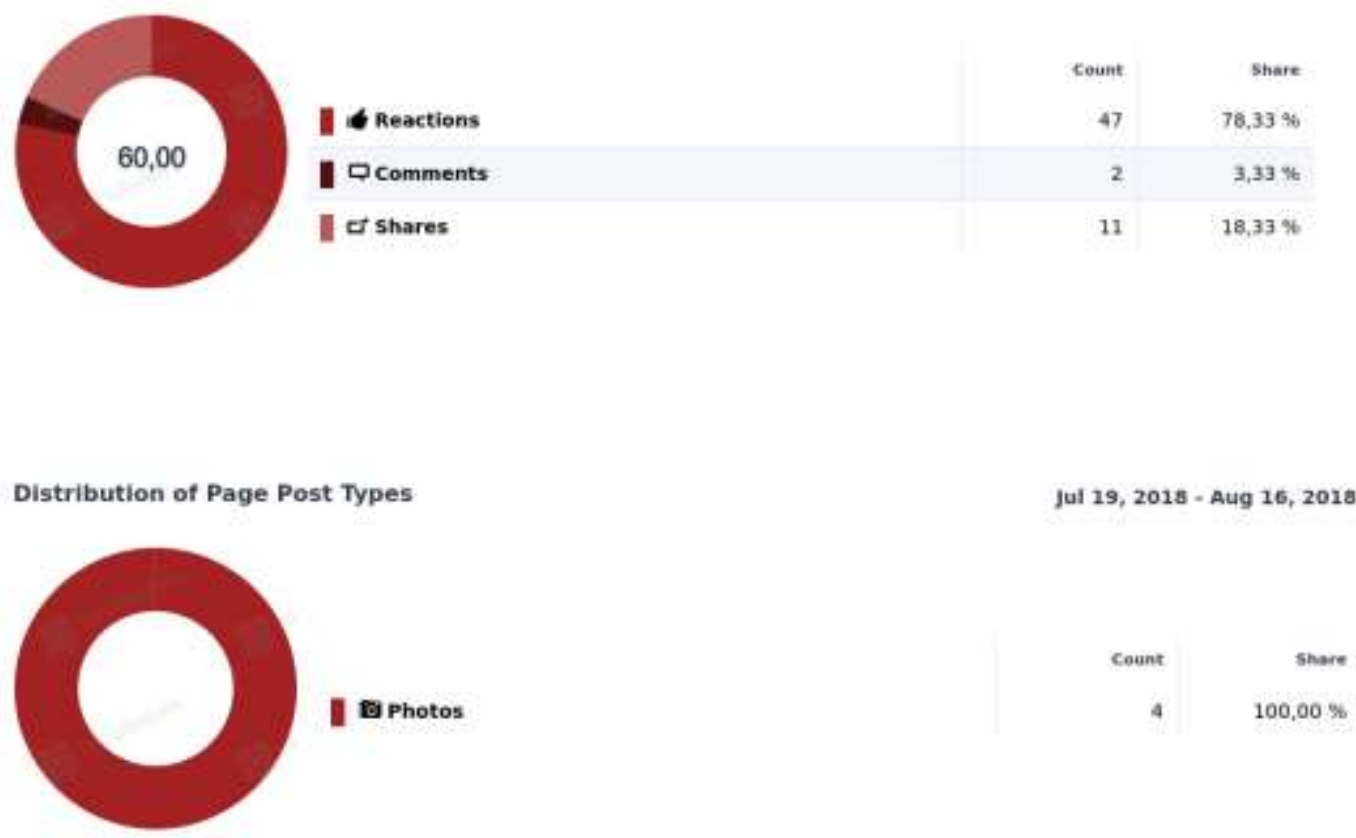

Most Engaging Post Types

Jul 19, 2018 - Aug 16, 2018

Avg litterations per 1000 Fans

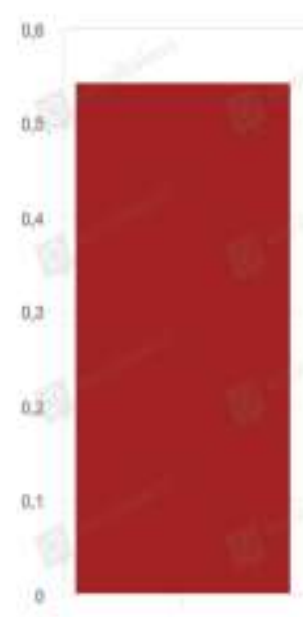

Holp

Man

sanua

Vised

othen

Figura 10. Facebook/Páginas de Instituciones Públicas del Ecuador.

Fuente: Social Baker \& autores a partir de la Superintendencia de Compañías. 


\section{Facebook Pages Stats in Ecuador}

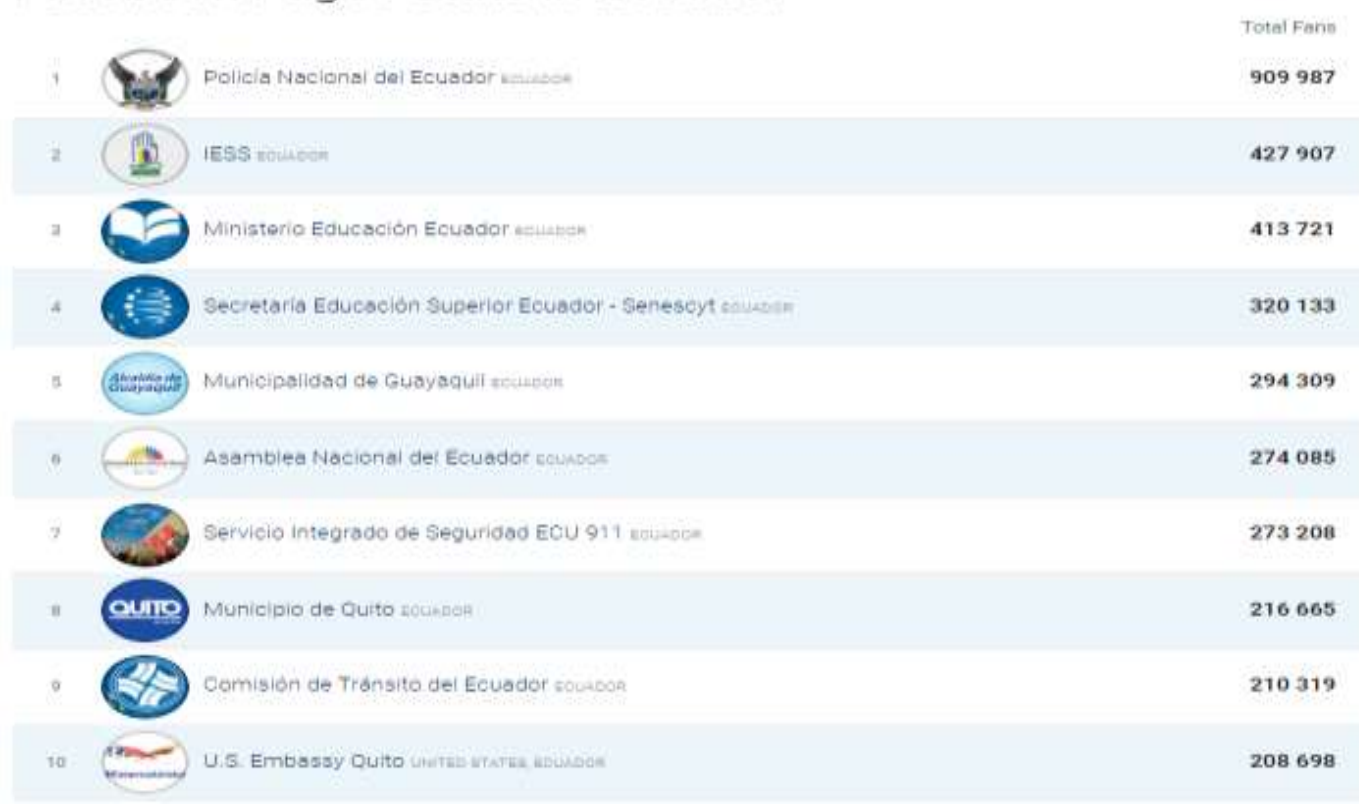

Figura 11. Facebook/Páginas de Instituciones Públicas del Ecuador

Fuente: Social Baker \& autores a partir de un análisis comparativo de Redes Sociales.

En el caso de Facebook, Policía Nacional del Ecuador encabeza la lista, sus inmediatos seguidores son IESS, Ministerio de Educación, Senescyt y Municipalidad de Guayaquil. También se pudo evidenciar que dentro del top ten no se encuentran instituciones relacionadas con el escenario productivo y comercial. De hecho, la Superintendencia de Compañías y el Ministerio de Industrias aparecen recién en la posición 49 y 51 , respectivamente. Por si fuera poco, el Ministerio de Turismo aparece en la posición 20, mientras que el MIES ocupa el puesto 25. Sorprendentemente, el Instituto Ecuatoriano de Economía Popular y Solidaria aparece en el lugar número 96.

Si se considera la gran influencia que tiene Facebook a nivel mundial y, sobre todo, en Ecuador, no se deduce otra cosa, sino que el posicionamiento online de las instituciones que conforman el sector mencionado no va por buen camino. Hay un indicador que es determinante, el Ministerio de Turismo no ocupa las primeras posiciones, entonces hay un problema de promoción, no solo a nivel nacional sino también internacional. En términos de desarrollo, la Economía Popular y Solidaria no tiene una gran interacción con la población, es decir, las personas no receptan la información necesaria para financiar sus ideas de negocio. Entonces surgen varios problemas para emprender o para asociarse. 


\section{Twitter Profiles Stats in Ecuador}

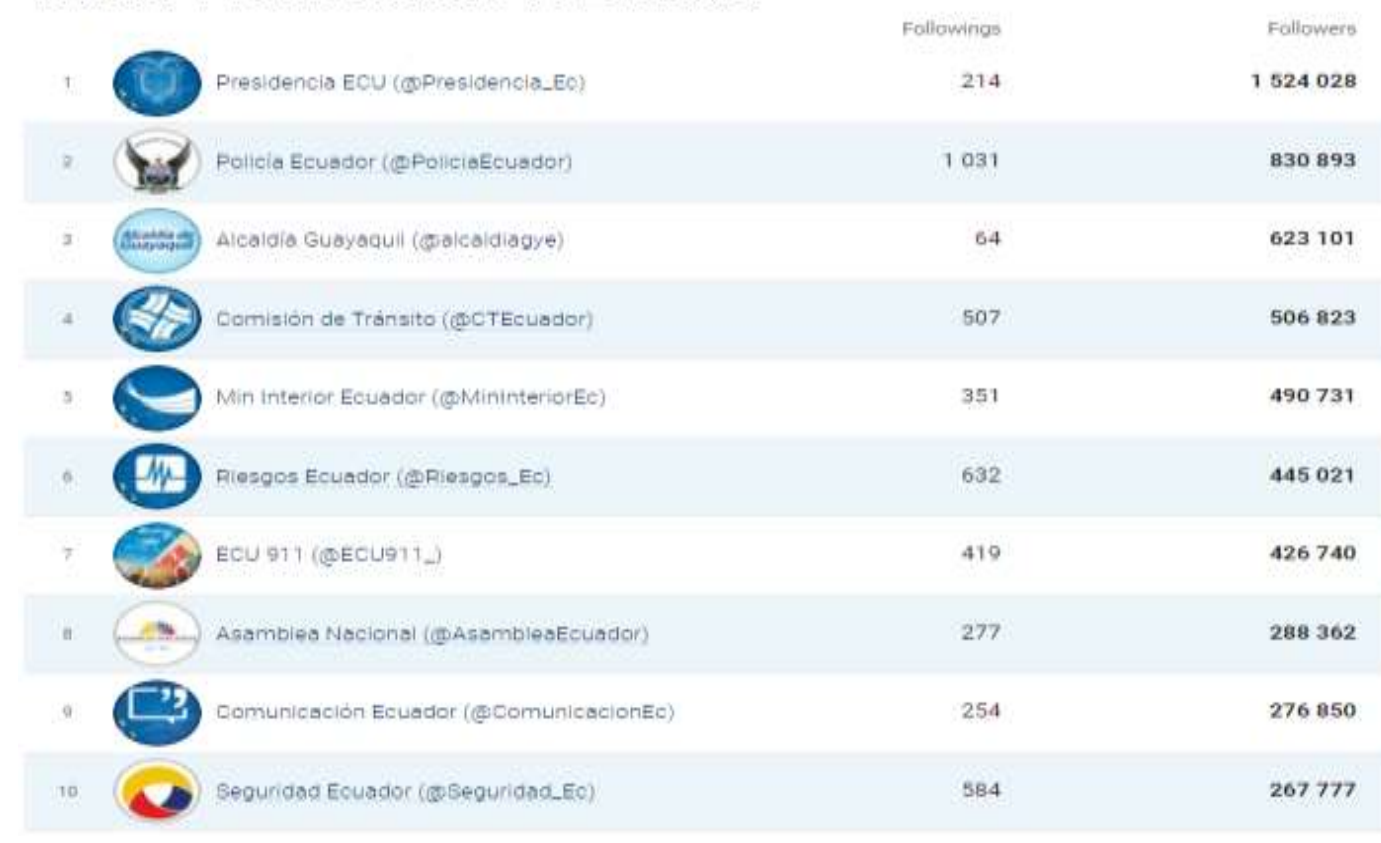

Figura 12. Twitter/Cuentas de Instituciones Públicas del Ecuador.

Fuente: Social Baker \& autores a partir de un análisis comparativo de Redes Sociales.

Las estadísticas de Twitter evidencian que la cuenta de la Presidencia del Ecuador encabeza la lista, sus inmediatos seguidores son Policía Nacional, Alcaldía de Guayaquil, Comisión de Tránsito y Ministerio del Interior. En esta red social, El Ministerio de Turismo ocupa la posición 13, una mejor ubicación que la cuenta de Facebook. El Ministerio de Industrias, Pro Ecuador y la Superintendencia de Compañías aparecen en la posición 35, 44 y 61, respectivamente. El Instituto de Economía Popular y Solidaria (IEPS) ocupa la casilla 63. Si bien es cierto, el posicionamiento en Twitter es mejor que en otras redes sociales, hay que destacar que la mayoría de los usuarios frecuentes poseen un nivel educativo más alto, lo cual podría ser una de las razones de su frecuente interacción

\section{YouTube Channels Stats in Ecuador}

$\begin{array}{rr}\text { Subscribers } & \text { Total uploaded video views } \\ 15992 & 10492119 \\ 12 & 10279\end{array}$




\section{Canales destacados}

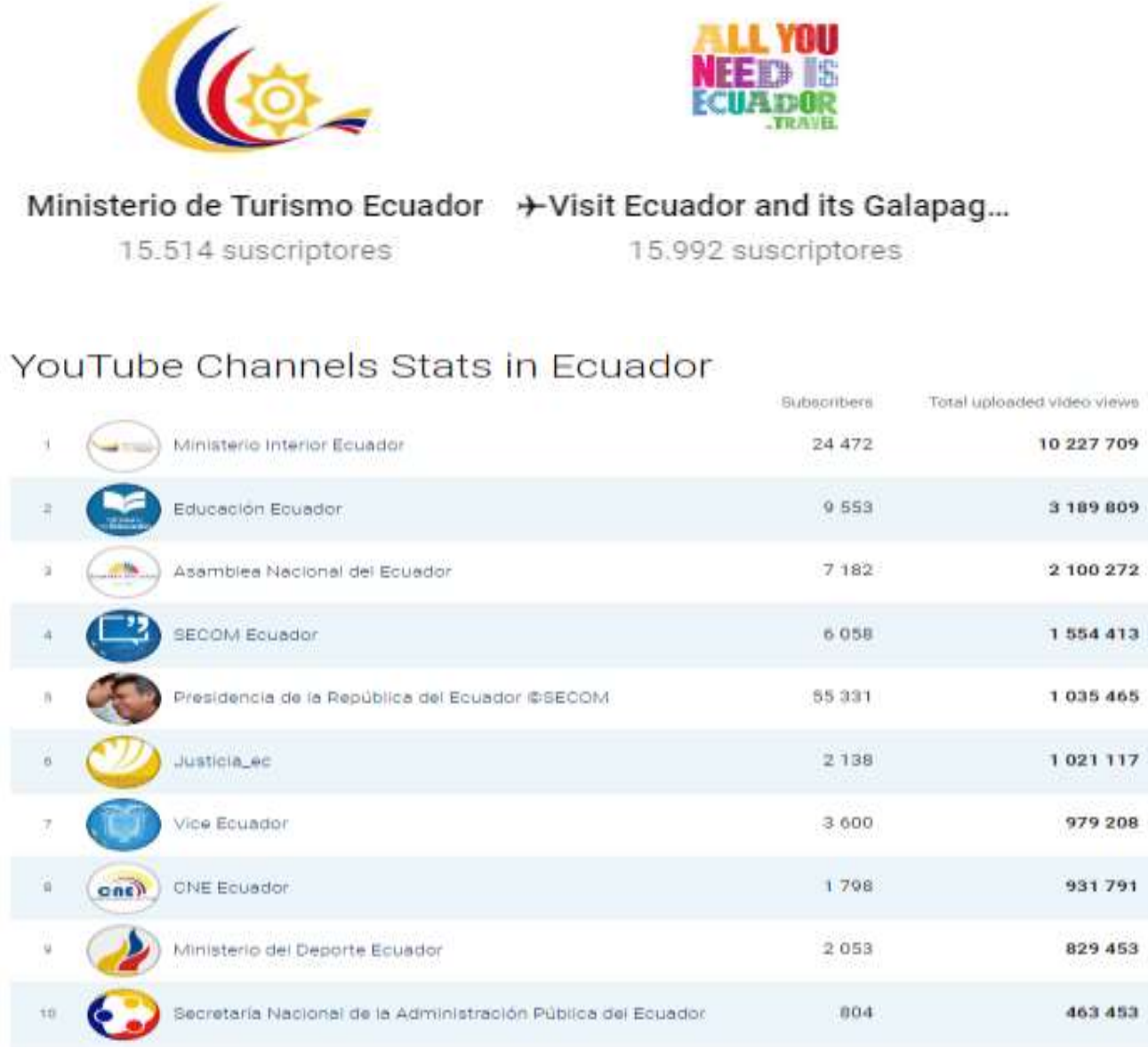

Figura 13. Youtube/Canales de Instituciones Públicas del Ecuador

Fuente: Social Baker \& autores a partir de un análisis comparativo de Redes Sociales.

Como se puede apreciar, el Ministerio de Turismo no es el canal rey en Youtube. Sin embargo, está ubicado en el top 5, en términos de suscriptores y total de vistas de video. De hecho, sus variantes, pertenecientes al mismo sector, ocupan casilleros nada despreciables. Por otro lado, se puede apreciar que el resto de instituciones objeto de estudio no están ubicadas ni siquiera entre las 10 primeras. 


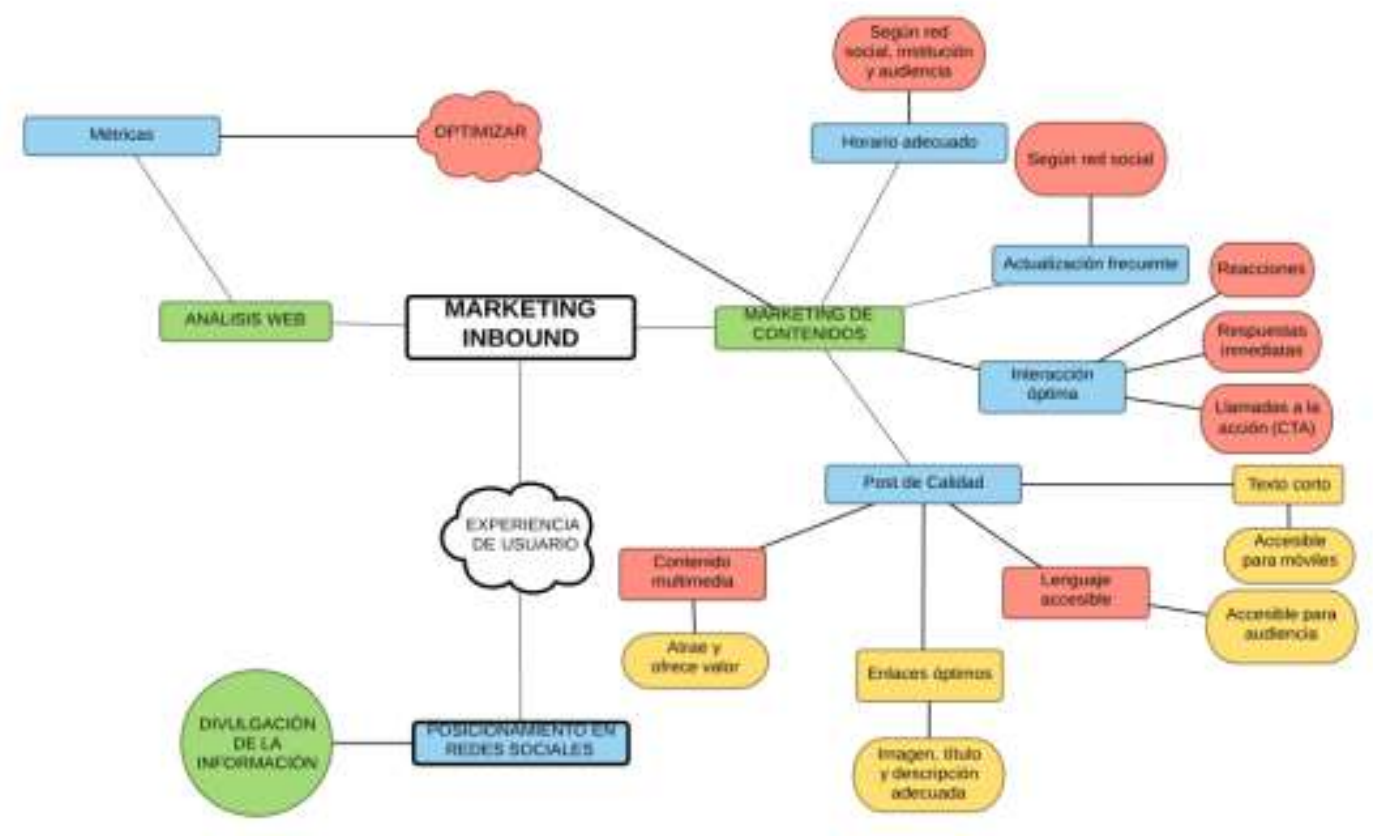

Figura 14. MODELO ESTÁNDAR para posicionar Instituciones Públicas en Redes Sociales.

Fuente: Elaboración propia.

\section{CONCLUSIONES Y RECOMENDACIONES}

La investigación ha permitido identificar los factores determinantes de posicionamiento en las redes sociales de las instituciones públicas del Ecuador, entre los cuales se destacan: experiencia de usuario y contenido publicado. Por consiguiente, la propuesta está enfocada en mejorar estas dos variables, puesto que ambas son influyentes en redes sociales. Es decir, la actividad frecuente de un usuario depende mucho del contenido que se genere en una cuenta. Entonces, el Marketing Inbound juega un rol vital para la visibilidad y alcance en redes sociales. Asimismo, su aplicación será complementada con el Marketing de Contenidos.

Las instituciones públicas que han sido analizadas presentan algunos problemas en términos de contenido, esto es: la información es relevante, pero al momento de difundirla, no logra el alcance deseado. Entonces, la sociedad civil no puede empoderarse del conocimiento, limitando la interacción y, sobre todo, la comunicación.

Ahora bien, la propuesta consiste en una guía cuyo objetivo principal es mejorar la divulgación de la información en redes sociales por parte de las instituciones públicas del Ecuador. Como se mencionó anteriormente, hay un problema al momento de generar contenido. Así pues, la 
siguiente ilustración es un modelo estándar donde se detallan los aspectos más importantes a tener en cuenta:

- Las instituciones públicas con bajo posicionamiento en redes sociales deben realizar un análisis web que extraiga todos los detalles sobre el comportamiento de los usuarios. Para ello, se recomienda utilizar una estrategia de Marketing Inbound, esto permitirá conocer las métricas y tomar decisiones en base a los resultados obtenidos en tiempo real. En esta etapa se mide, se analiza y se identifica variables influyentes En este caso, SocialBakers es una plataforma ideal para medir redes sociales.

- Una campaña de Marketing Inbound es de tipo orgánico, esto es, no supone costos de publicidad para alcanzar audiencia. Para lograrlo, utiliza otra estrategia conocida como Marketing de Contenidos, dado que el tipo de contenido es vital para fidelizar clientes, se fundamenta en mejorar la experiencia de usuario. Es decir, el contenido y la forma cómo se publica permiten atraer audiencia, crear valor y difusión de la información. Este tipo de campañas no es intrusivo, es totalmente democrática y muy efectiva. En esta etapa, se selecciona el tipo de estrategia basada en la variable dependiente más afectada en el análisis de datos. En este caso, se comprobó que el contenido es determinante.

- Una estrategia de Marketing de Contenidos se enfoca en todas las aristas que pueden afectar al contenido publicado. En esta etapa se diseña el contenido que aumentará la visibilidad web, en términos de alcance e impresión. Así, por ejemplo:

\section{Horario:}

Las publicaciones deben regirse a los horarios de cada red social, es decir, hay momentos del día en el que hay mayor número de usuarios conectados, ya sea en Facebook (09-15h00), Twitter (12-15h00) o YouTube. Asimismo, hay que tener en cuenta el horario de cada institución, esto supondrá un carácter formal y oficial a cada post. A pesar de que es difícil saber con exactitud el momento en el cual el público objetivo está conectado, es posible estimarlo, así se elevará la probabilidad de replicar la información. No solo tendrá más alcance, sino también más impresiones. En otras palabras, si la información llega a las personas principalmente interesadas, ellas se encargarán de difundirla, independientemente de si la audiencia esté o no interesada. 


\section{Actualización:}

Hay que evitar la monotonía. Si hay abundancia de publicaciones, perderán valor. La frecuencia con la que se publica debe ser adecuada. Para Facebook, se recomienda entre 1-3 posts por día. En Twitter se sugiere 3-5 tweets por día y para YouTube 1-3 clips por semana.

\section{Post:}

-Redacción: el texto debe ser corto, puesto que el entorno de las redes sociales es inmediato. La mayoría de usuarios no espera un texto largo. Además, los textos breves facilitan la lectura desde los smartphones, dado que la mayor parte de la audiencia revisa las redes sociales desde este tipo de dispositivos. Asimismo, para generar confianza, la redacción no debe tener faltas ortográficas. Si las hay, pierde el carácter de oficial.

-Lenguaje: debe evitarse los tecnicismos, el objetivo siempre será que la sociedad civil se empodere del conocimiento. Para ello, la información debe ser comunicada utilizando un lenguaje accesible.

-Tipo de contenido: estadísticamente hablando, el tipo de contenido que más atrae audiencia es el multimedia. Crea valor y genera interés entre los usuarios, principalmente las infografías. Utilizar el storytelling para informar de una forma puntual y concisa aumenta la visibilidad web. Los clips son determinantes, sobre todo, aquellos que tienen una duración breve e información puntual.

-Enlaces: siempre deben estar acompañados de una imagen en miniatura, ésta debe ser adecuada. Deben incluir título y descripción breve, as Estas tres condiciones son determinantes de la calidad de un enlace. También es importante que el enlace sea corto, puesto que facilitará la replicación del mismo.

\section{Interacción:}

Los CTA (llamadas a la acción) son muy útiles a la hora de la conversión. Esta es una condición influyente para la audiencia. Proporciona identidad y sentido de pertenencia. Indudablemente, los usuarios esperan participar, sentirse parte de la comunidad y reaccionar por medio de un click o un post. Para consolidar la interacción, es importante que el perfil institucional humanice las respuestas y que la entrega sea inmediata.

- SocialBakers es una plataforma ideal para medir redes sociales en tiempo real. Es muy útil para hacer remarketing y analizar los datos después de la implementación de 
las estrategias de contenidos. Se revisan dos variables: visibilidad y posicionamiento en redes sociales, ambas son determinantes de la experiencia de usuario. Por consiguiente, si mejora la experiencia de usuario, mejora la divulgación de información desde las instituciones públicas en redes sociales.

\section{REFERENCIAS BIBLIOGRÁFICAS}

Andrade, D. (2016). Estrategias de marketing digital en la promoción de Marca Ciudad. Revista Escuela de Administración de Negocios, (80), pp. 69

Araujo, G. (2016). Elementos del inbound marketing para optimizar el valor del cliente sucrense de las empresas telefónicas. Investigación \& Negocios, pp. 12

Barrientos, P. (2017). Marketing + internet $=$ e-commerce: oportunidades y desafíos. Revista Finanzas y Política Económica, 9(1), pp. 43

Criado, J. I. (2016). "Redes Sociales y Administraciones Públicas. Hacia una gestión estratégica en el sector público", en J. R. Gil-García, J. I. Criado y J. C. Téllez (eds). Tecnologías de Información y Comunicación en la Administración Pública: Conceptos, Enfoques, Aplicaciones y Resultados,México, INFOTEC, pp. 227-260.

Codina, LI.; Gonzalo, C.; Pedraza, R.; Rovira, C. (2017). Posicionamiento Web y Medios de Comunicación: Ciclo de Vida de una Campaña y Factores. 